\title{
ATIVIDADE ELETROCATALÍTICA DE SISTEMAS BIOMIMÉTICOS DA ENZIMA CATALASE
}

\author{
Iorquirene de Oliveira Matos e Wendel A. Alves* \\ Centro de Ciências Naturais e Humanas, Universidade Federal do ABC, 05580-970 Santo André - SP / Instituto Nacional de \\ Ciência e Tecnologia de Bioanalítica, CP 6154, 13083-970 Campinas - SP, Brasil \\ Otaciro R. Nascimento \\ Centro de Ciências Naturais e Humanas, Universidade Federal do ABC, 05580-970 Santo André - SP / Departamento de Física e \\ Informática, Instituto de Física de São Carlos, Universidade de São Paulo, 13565-905 São Carlos - SP, Brasil
}

Recebido em 2/12/10; aceito em 4/5/11; publicado na web em 8/7/11

\begin{abstract}
ELECTROCATALYTIC ACTIVITY OF CATALASE BIOMIMETIC SYSTEMS. In this work, we describe the immobilization of the dinuclear compound $\left.\left[\mathrm{Cu}_{2} \text { (apyhist }\right)_{2} \mathrm{Cl}_{2}\right]\left(\mathrm{ClO}_{4}\right)_{2}(\mathbf{1})$ and its derived cations complexes, obtained in water solution or by deprotonation of the imidazolate moiety in the ligand leading to a cyclic tetranuclear species, in the $\mathrm{Nafion}^{\circledast}$ membrane on glass carbon electrode surface. After that, we studied the influence of the equilibrium in the electrocatalytic activity towards the reduction of $\mathrm{H}_{2} \mathrm{O}_{2}$ in the development of an amperometric sensor for the analytical determination of hydrogen peroxide. This strategy proved successful, and the electrochemical behaviour of the all complexes formed within the $\mathrm{Nafion}^{\circledast}$ coatings was characterized. We also provide evidence that its related cyclic tetranuclear imidazolate-bridged complex acts as a catalysts for the intramolecular, two-electron reduction of $\mathrm{H}_{2} \mathrm{O}_{2}$.
\end{abstract}

Keywords: enzymeless biosensors; copper(II) complexes; bioinorganic chemistry.

\section{INTRODUÇÃO}

A tecnologia dos biossensores é um campo aberto para inovação e desenvolvimento de novos dispositivos eletroquímicos de interesse tecnológico. Os estudos para elucidar e construir mecanismos de transferência eletrônica, direta ou mediada, é indispensável para o sucesso dos biossensores na tecnologia do futuro. ${ }^{1}$ Além disso, são importantes, também, para a compreensão dos princípios fundamentais de reconhecimento biológico e a comunicação entre a enzima e o transdutor. ${ }^{2} \mathrm{O}$ mecanismo de transferência eletrônica direta neste tipo de sistema ainda não é bem conhecido. Existem ainda várias questões a serem esclarecidas, tais como, quais são os parâmetros (estruturais e cinéticos) que determinam a possibilidade da enzima redox catalisar uma reação no eletrodo; qual é o papel da enzima proteica na eletrocatálise e no processo de transferência eletrônica; qual é a influência da natureza do material e da estrutura na superfície do eletrodo; qual é a relação entre o mecanismo catalítico da enzima e a capacidade de transferência eletrônica direta. ${ }^{3}$ Estudos são fundamentais para a síntese de novos materiais para o desenvolvimento de bioeletrocatalisadores que sejam altamente eficazes e permitam a geração de novos dispositivos eletrônicos.

Nessa busca constante na melhora da seletividade e sensibilidade de resposta, surge o desenvolvimento de uma nova área de sensores amperométricos baseados em sistemas biomiméticos com o intuito de promover um aumento na transferência eletrônica entre eletrodo/sítio ativo/substrato, aumentando a sensibilidade do sistema e facilitando a construção de eletrodos modificados que determinem substâncias biológicas de interesse analítico, podendo ser de importância clínica, ambiental ou industrial. ${ }^{4} \mathrm{O}$ desempenho analítico de sensores biomiméticos, em alguns casos, mostra-se superior em relação aos correspondentes biossensores enzimáticos. ${ }^{5}$

\footnotetext{
*e-mail: wendel.alves@ufabc.edu.br
}

Compostos biomiméticos são resultado do estudo da formação, estrutura ou função de substâncias biologicamente produzidas e materiais (enzimas ou seda) e os mecanismos e processos biológicos (síntese de proteínas ou fotossíntese) especialmente para o propósito de sintetizar produtos similares por meio de mecanismos artificiais que imitem os naturais. ${ }^{6,7}$

No entanto, mimetizar estruturas biológicas nem sempre é fácil. Estruturas nem sempre funcionam da mesma forma, pois se adaptam às exigências funcionais locais. Mesmo as plantas e animais mais simples podem sentir o mundo, integrar informações e agir em conformidade com as necessidades. A construção de um sensor biomimético é um campo promissor na área de desenvolvimento de procedimentos analíticos para determinação de diferentes analitos. $^{8}$

Um fator essencial para o desenvolvimento destes sensores está baseado em controlar a transferência de elétrons entre o reagente que efetua o reconhecimento catalítico, aquele que faz a função do sítio ativo da enzima na superfície do eletrodo. A construção deste tipo de sensores está focada na estrutura do sítio ativo da enzima, que será utilizada como base para a determinação do substrato de interesse, e o mecanismo da reação que será catalisada. Neste contexto, vale a pena ressaltar os excepcionais avanços na área de manipulação de estruturas biológicas, de tal forma que certas proteínas com atividade redox, em que novos sítios adequados para a inserção de metais são introduzidos, e ácidos nucleicos/DNA modificados estão se tornando modelos versáteis para a melhor elucidação das funções biológicas. Paralelamente aos estudos de biomiméticos, outro assunto de fundamental importância está relacionado ao desenvolvimento de sistemas modelos funcionais que são moléculas mais simples e que podem ser utilizados para elucidar o mecanismo de ação de certas proteínas ou enzimas, além de sua potencial aplicação como novos catalisadores de interesse industrial. Diversos complexos de metais de transição podem ser utilizados como sistemas modelo. A literatura relata a 
utilização de complexos de cobre(II) no desenvolvimento de sensores para determinação das catequinas em chá verde, ${ }^{9}$ hidroquinona em $\operatorname{cosméticos},{ }^{8}$ dopamina em fármacos, ${ }^{10}$ entre outros. Estudos utilizando complexos de ferro incluem a determinação eletroanalítica de pirodoxina (vitamina $\mathrm{B}_{6}$ ), ${ }^{11}$ da substância oncocalixona ${ }^{12}$ e compostos fenólicos. ${ }^{13}$ Complexos de manganês foram estudados para análise de rutina ${ }^{14}$ e fenóis. ${ }^{15} \mathrm{Um}$ complexo de ródio foi estudado para determinação de hidrazina. ${ }^{16}$

Neste trabalho estudou-se a construção de sensores biomiméticos da enzima catalase, que é encontrada em organismos aeróbicos que vão desde bactérias a plantas e animais. ${ }^{17}$ Estas enzimas apresentam duas funções: catalítica - com a decomposição de peróxido de hidrogênio em água e oxigênio e peroxidativa - leva as toxinas e $\mathrm{H}_{2} \mathrm{O}_{2}$, potencialmente nocivos, a recombinar-se para produzir água e produtos inofensivos ou úteis, tais como, alcoóis, fenóis, aldeídos e ácidos. ${ }^{18}$ Desta maneira, as enzimas catalases protegem os organismos vivos de espécies reativas de oxigênio que são responsáveis pelo estresse oxidativo, que são reações na célula que levam, por exemplo, ao envelhecimento celular, diabetes e câncer. ${ }^{19-22}$

Dessa forma, a detecção de peróxido de hidrogênio vem recebendo atenção na pesquisa de sensores nas últimas décadas. Muitas técnicas são usadas para detecção de $\mathrm{H}_{2} \mathrm{O}_{2}$ em produtos químicos, amostras biológicas, clínicas, ambientais e muitas outras estão em desenvolvimento. ${ }^{22-25}$ Entre elas, a detecção amperométrica é um campo promissor para detecção rápida, precisa e seletiva de peróxido de hidrogênio. ${ }^{22,26-30}$

Diante disso, estudou-se um sensor biomimético da enzima catalase, utilizando um complexo de cobre(II) contendo ligantes tridentados do tipo base Schiff e um grupo imidazol imobilizado em membrana Nafion ${ }^{\circledR}$ sobre a superfície de um eletrodo de carbono vítreo (ECV). Esse complexo possui a vantagem de apresentar um sistema redox bem definido, estável em meio fisiológico, com boa reprodutibilidade e sensibilidade. ${ }^{31}$ Em solução aquosa, este complexo forma duas espécies mono- e tetranucleares de cobre(II) que estão em equilíbrio em solução aquosa, onde os centros de cobre estão ligados por meio de um ligando ponte imidazolato (Esquema 1). ${ }^{32-37}$ Estudos eletroanalíticos de detecção amperométrica frente ao peróxido de hidrogênio indicaram que as espécies tetranucleares são melhores eletrocatalisadores do que os compostos de cobre(II) mononucleares.

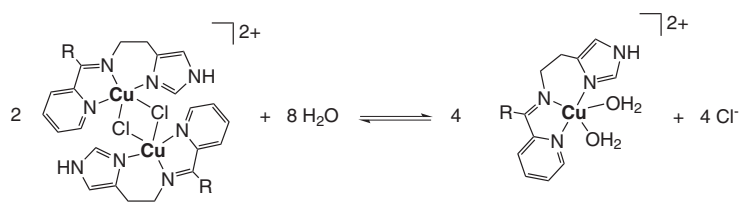

$\left[\mathrm{Cu}_{2} \text { (apyhist) }{ }_{2} \mathrm{Cl}_{2}\right]^{2+}$ (1), $\mathrm{R}=\mathrm{CH}_{3}$

$\left[\mathrm{Cu}_{2}(2-\text { pyhist })_{2} \mathrm{Cl}_{2}\right]^{2+}$ (2), $\mathrm{R}=\mathrm{H}$
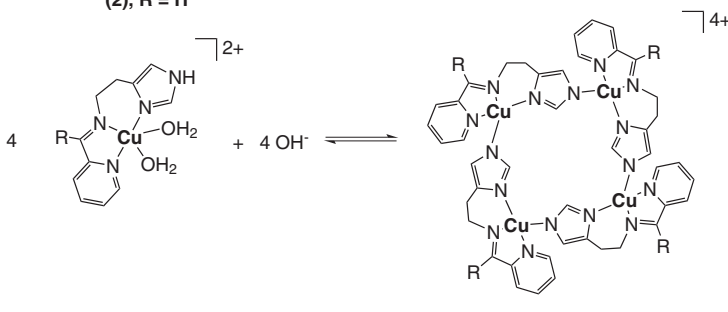

$+12 \mathrm{H}_{2} \mathrm{O}$

$\left[\mathrm{Cu}_{4}(\text { apyhist })_{4}\right]^{4+}$

(3), $\mathrm{R}=\mathrm{CH}_{3}$

$\left[\mathrm{Cu}_{4}(2-\text { pyhist })_{4}\right]^{4+}$

(4), $\mathrm{R}=\mathrm{H}$
Complexos com estruturas cíclicas parecem facilitar a interação do substrato com o sítio ativo do complexo. Neste trabalho a quantidade de íons cobre utilizada foi sempre a mesma, com variações apenas no ligante ao redor do íon metálico, o que levou a alterações significativas na sensibilidade dessas espécies frente à detecção do peróxido de hidrogênio.

\section{PARTE EXPERIMENTAL}

\section{Reagentes}

Todos os reagentes empregados foram de grau e pureza analítica e utilizados sem purificação prévia. Fosfato de potássio dibásico tri-hidratado, fosfato de potássio monobásico, cloreto de cobre(II) di-hidratado, dicloridrato de histamina, 2-acetilpiridina e perclorato de sódio foram de procedência da Sigma-Aldrich. Cloreto de potássio, hidróxido de potássio, ácido clorídrico e peróxido de hidrogênio $29 \%$, assim como os solventes alcoóis etílico e metílico foram de procedência da Labsynth. Perclorato de cobre(II) foi de procedência da Acros Organics e Nafion ${ }^{\circledR} 5 \%$ foi de procedência da DuPont.

\section{Equipamentos}

Os eletrodos utilizados foram carbono vítreo como trabalho $\left(0,062 \mathrm{~cm}^{2}\right)$ da Metrohm- Pensalab, platina como auxiliar e eletrodo de calomelano saturado (ECS) como referência. As medidas voltamétricas e amperométricas foram realizadas em potenciostato/ galvanostato $\mu$ Autolab Fra 2, Type III. As medidas de $\mathrm{pH}$ foram efetuadas com um pHmetro Metrohm - Pensalab, modelo 827 pH lab, acoplado a um eletrodo de vidro combinado, também da Metrohm - Pensalab. A calibração do eletrodo de vidro foi feita antes do preparo das soluções, com soluções tampões adequadas. As medidas de absorção na região do visível foram efetuadas num espectrofotômetro Varian, modelo Cary 50. Foram empregadas celas usuais retangulares de quartzo de caminho óptico igual a $1 \mathrm{~cm}$, utilizando faixa de 200 a $800 \mathrm{~nm}$. O sistema de rotaevaporação utilizado na preparação dos complexos de cobre(II) foi de procedência da Buchi-Pensalab, modelo Rotavapor R-210 e o banho refrigerado de procedência da Solab Científica. A limpeza dos eletrodos foi realizada em banho de ultrassom da Sanders, modelo UltraSonic Cleaner - SW2000FI, com aquecimento.

\section{Limpeza dos eletrodos}

Antes do preparo de cada eletrodo, o seguinte procedimento de limpeza do ECV (eletrodo de carbono vítreo) foi seguido: limpeza em pano de polimento NTR Ø $200 \mathrm{~mm}$ de uso geral abrasivo $<1 \mu \mathrm{m}$ com suspensão de alumina $1 \mu \mathrm{m} n^{\circ}$. 4; lavagem com água desionizada; ultrassom por $15 \mathrm{~min}$, imerso em álcool etílico e lavagem com água desionizada.

\section{Preparação e caracterização dos complexos de cobre(II)}

Perclorato de bis( $\mu$-cloro)(4-imidazolil)etileno-2-amino-1etilpiridina) de cobre(II), $\left.\left[\mathrm{Cu}_{2} \text { (apyhist }\right)_{2} \mathrm{Cl}_{2}\right]\left(\mathrm{ClO}_{4}\right)_{2}(\mathrm{I})$

Este composto dinuclear de cobre(II) foi preparado utilizando a metodologia descrita na literatura, ${ }^{34}$ com pequenas variações no procedimento experimental.

Adicionou-se, na capela e sob agitação, $736 \mathrm{mg}(4 \mathrm{mmol})$ de dicloridrato de histamina a uma mistura de $740 \mathrm{mg}(2 \mathrm{mmol}) \mathrm{de}$ perclorato de cobre(II), dissolvidos em $10 \mathrm{~mL}$ de água desionizada e $50 \mathrm{~mL}$ de álcool metílico. Após completa homogeneização da mistura, adicionou-se, gota a gota, $244 \mathrm{mg}$ (2 mmol) de 2-acetilpiri- 
dina dissolvidos em $15 \mathrm{~mL}$ de álcool metílico. A reação foi deixada sob agitação constante à temperatura ambiente, durante $2 \mathrm{~h}$. Em seguida, acondicionou-se a solução final obtida no próprio balão de reação em geladeira, até precipitação dos cristais do complexo de cobre(II). Filtrou-se o precipitado obtido, de cor verde e, finalmente, secou-se num dessecador a vácuo. Rendimento: 68\%. Análise elementar, calculado para $\left[\mathrm{Cu}_{2} \mathrm{~N}_{8} \mathrm{C}_{24} \mathrm{H}_{28} \mathrm{Cl}_{2}\right]\left(\mathrm{ClO}_{4}\right)_{2}(\mathrm{MM}=825,4 \mathrm{~g} / \mathrm{mol})$ : $\% \mathrm{C}=34,92, \% \mathrm{H}=3,42, \% \mathrm{~N}=13,57$; obtido: $\% \mathrm{C}=34,78, \% \mathrm{H}=3,40$, $\% \mathrm{~N}=13,53$.

\section{Outros compostos de cobre(II) utilizados}

Para fins comparativos de estudo da influência do ligante ao redor do íon metálico na eletrocatálise da redução do $\mathrm{H}_{2} \mathrm{O}_{2}$, foram utilizados e sintetizados os seguintes complexos de cobre(II) já descritos na literatura: ${ }^{33,35,37} \mathrm{CuCl}_{2} \times 2 \mathrm{H}_{2} \mathrm{O},\left[\mathrm{Cu}_{2}(2 \text {-pyhist })_{2} \mathrm{Cl}_{2}\right]\left(\mathrm{ClO}_{4}\right)_{2}(2)$, $\left.\left[\mathrm{Cu}_{4} \text { (apyhist) }\right)_{4}\right]\left(\mathrm{ClO}_{4}\right)_{4}(3)$ e $\left.\left[\mathrm{Cu}_{4}(2 \text {-pyhist })_{4}\right)\right]\left(\mathrm{ClO}_{4}\right)_{4}$ (4), onde: 2-pyhist $=(4$-imidazolil)etileno-2-aminometilpiridina .

\section{Modificação dos eletrodos}

Para a modificação dos eletrodos de carbono vítreo envolvendo os complexos de cobre(II) com a membrana de Nafion ${ }^{\circledR}$, pesou-se aproximadamente $20 \mu \mathrm{mol}$ em relação ao íon de cobre(II) dos complexos (1), (2), (3), (4) e $\mathrm{CuCl}_{2} \times 2 \mathrm{H}_{2} \mathrm{O}$ e dissolveu-se em 200 $\mu \mathrm{L}$ de Nafion ${ }^{\circledR} 5 \%$, respectivamente. A seguir, adicionou-se 1000 $\mu \mathrm{L}$ de água desionizada (em pH 7,0 ou 9,0) e levou-se a mistura ao aparelho de ultrassom, até completa homogeneização. As soluções foram armazenadas em geladeira. Em seguida, foram adicionados $4,5 \mu \mathrm{L}$ desta mistura à superfície do eletrodo, deixando-se secar a temperatura ambiente por $1 \mathrm{~h}$, formando-se um filme híbrido na superfície do eletrodo. Posteriormente, os eletrodos foram hidratados, por $10 \mathrm{~min}$, em eletrólito tampão fosfato, o mesmo usado para realizar as medidas de voltametria cíclica e amperometria. $\mathrm{O}$ eletrólito permaneceu sob atmosfera de gás nitrogênio, por 10 min, antes do início das medidas. Medidas com ECV sem modificação (eletrodo branco) foram realizadas com o objetivo de comparar os resultados.

\section{Detecção do peróxido de hidrogênio}

Realizaram-se medidas amperométricas para verificar a sensibilidade do eletrodo modificado frente à detecção do peróxido de hidrogênio, onde foram adicionados, sob agitação constante, $10 \mu \mathrm{L}$ de solução de $\mathrm{H}_{2} \mathrm{O}_{2}\left(0,01 \mathrm{~mol} \mathrm{~L}^{-1}\right)$ em célula de $8 \mathrm{~mL}$, que corresponde à adição de $12,5 \mu \mathrm{mol} \mathrm{L}^{-1}$ de peróxido de hidrogênio, em cada adição. Realizaram-se, em média, 10 adições sucessivas em cada medida da resposta amperométrica na redução do $\mathrm{H}_{2} \mathrm{O}_{2}$.

\section{RESULTADOS E DISCUSSÃO}

Diferentes complexos de cobre(II) contendo um ligante tridentado do tipo base de Schiff além de um grupo imidazol foram preparados, na forma de sais de perclorato, e caracterizados através de diferentes técnicas analíticas e espectroscópicas. ${ }^{33,35,37}$ Estes compostos já haviam sido estudados anteriormente e tiveram suas estruturas determinadas por cristalografia de raios X.$^{35}$ Estudos prévios mostraram que em solução aquosa existe a labilidade dos íons cloreto para os complexos (1) e (2) e a respectiva formação da espécie mononuclear, conforme mostrado no Esquema 1. Adicionalmente, foi também observado que em solução aquosa, a pH elevados, estes complexos estão em equilíbrio com a correspondente espécie tetranuclear (3) e (4), na qual os centros de cobre estão ligados por um ligante ponte imidazolato. ${ }^{32-37}$
Caracterização voltamétrica para o eletrodo modificado com a espécie (1) em membrana Nafion ${ }^{\circledR}$ em diferentes valores de pH

Estudos de voltametria cíclica em diferentes velocidades de varredura foram realizados para o eletrodo modificado com o complexo (1) na presença de membrana Nafion ${ }^{\circledR}$, conforme mostrado na Figura 1. Particularmente, o polímero Nafion ${ }^{\circledR}$ foi escolhido devido à presença dos grupos sulfonatos $\left(-\mathrm{SO}_{3}{ }^{-}\right)$que promovem a imobilização da espécie catiônica sobre a superfície do eletrodo, devido à substituição do contraíon perclorato do complexo de cobre(II) pelo grupos $-\mathrm{SO}_{3}{ }^{-}$do polímero.

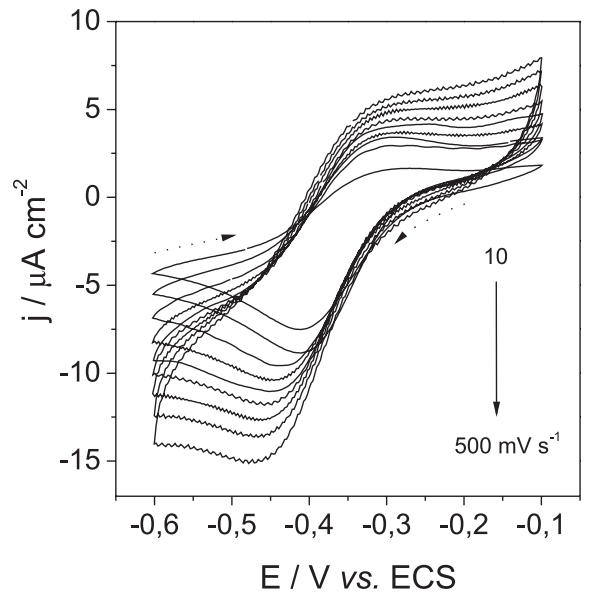

Figura 1. Estudo do efeito da velocidade de varredura para o ECV modificado com o complexo (1) em membrana de Nafion ${ }^{\circledR}$ em solução tampão fosfato pH 7,0 (0,1 $\left.\mathrm{mol} \mathrm{L}^{-1}\right)$

Os voltamogramas representados na Figura 1 apresentam dois picos, sendo um anódico em $-0,45 \mathrm{~V}$ e outro catódico em - $-0,40 \mathrm{~V}$

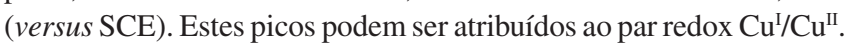
A existência de uma linearidade entre as correntes de pico redox e a raiz quadrada da velocidade de varredura indica que o processo é controlado por difusão.

\section{Atividade eletrocatalítica frente à detecção do peróxido de hidrogênio}

Os resultados mostram um significativo aumento na corrente de redução com adição de peróxido hidrogênio e deslocamento do potencial para valores menos negativos para o eletrodo modificado com o complexo (1), em relação ao ECV, conforme ilustrado na Figura $1 \mathrm{~S}$, material suplementar. Medidas amperométricas aplicando-se o potencial de $-0,50 \mathrm{~V}$ para o eletrodo modificado com adição de 10 $\mu \mathrm{L}$ de uma solução de $\mathrm{H}_{2} \mathrm{O}_{2}\left(10 \mathrm{mmol} \mathrm{L}^{-1}\right)$ mostram uma sensibilidade de resposta de $360 \mathrm{~mA} \mathrm{~cm}$ mol$^{-1} \mathrm{~L}$ (Figura 2). Estes estudos sugerem que estes compostos eletrocatalisam a redução do peróxido de hidrogênio, podendo ser utilizados como mediadores redox no preparo de sensores amperométricos. Neste caso, sugere-se o seguinte mecanismo de reação na superfície do eletrodo modificado: com a adição do analito ao eletrólito, $\mathrm{o}_{2} \mathrm{H}_{2}$ é reduzido a $\mathrm{H}_{2} \mathrm{O}$, por um mecanismo envolvendo dois elétrons, na superfície do eletrodo. Consequentemente, o complexo de cobre é oxidado, sendo regenerado a cobre(I) com aplicação de um potencial de $-0,50 \mathrm{~V}$ versus ECS. $^{32}$

\section{Estudo da variação do potencial aplicado e valores de $\mathrm{pH}$}

Os estudos mostram que a resposta do sensor é afetada pela aplicação de diferentes potenciais na faixa de 0 a - $0,50 \mathrm{~V}$. Neste caso, observa-se um aumento da sensibilidade com a diminuição do poten- 


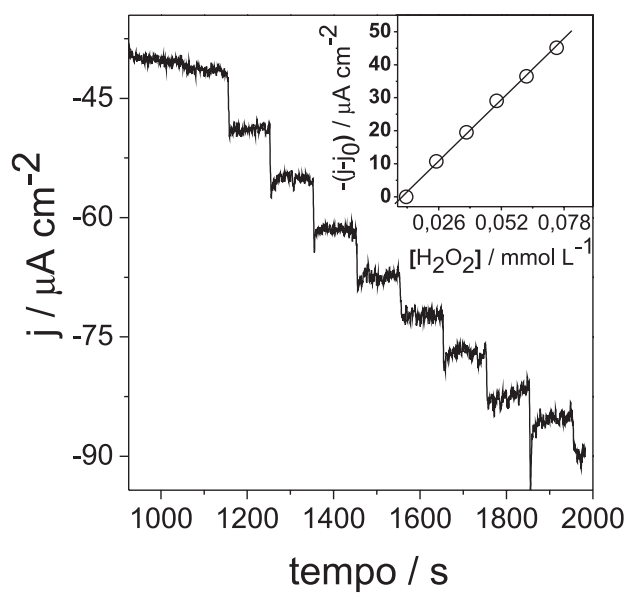

Figura 2. Resposta amperométrica em solução tampão fosfato pH 7,0 (0,1 mol $\left.\mathrm{L}^{-1}\right)$, potencial $-0,50 \mathrm{~V}$. Incremento de $12,5 \mathrm{mmol} \mathrm{L}^{-1}$ de concentração de $\mathrm{H}_{2} \mathrm{O}_{2}$, sob agitação magnética

cial, mostrando que a redução do $\mathrm{H}_{2} \mathrm{O}_{2}$ é favorecida em potenciais mais negativos (Figura 3). Os resultados mostram que o potencial ótimo de redução do $\mathrm{H}_{2} \mathrm{O}_{2}$ está em $-0,50 \mathrm{~V}$, que resultou na maior sensibilidade encontrada, e está em concordância com o potencial de redução completa de íons de cobre(II) sobre a superfície do eletrodo modificado, conforme observado no voltamograma da Figura 1 em solução tampão fosfato ( $\mathrm{pH} 7,0)$.

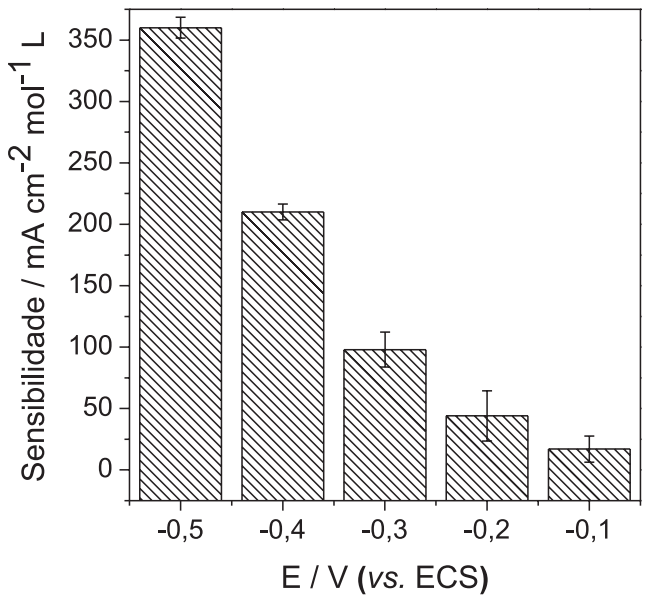

Figura 3. Estudo comparativo da influência do potencial nas sensibilidades obtidas frente à resposta amperométrica do peróxido de hidrogênio, medidas em solução tampão fosfato $\left(0,1 \mathrm{~mol} \mathrm{~L}^{-1}\right)$ e $\mathrm{pH}$ 7,0

Numa etapa posterior, estudou-se a influência da variação do $\mathrm{pH}$ do meio na resposta do sensor (Figura 4). As maiores sensibilidades foram obtidas nas faixas de $\mathrm{pH}$ entre 7,0 e 9,0, o que indica que a catálise é favorecida para o complexo tetranuclear (3) sobre o ECV imobilizado com membrana de Nafion ${ }^{\circledR}$, uma vez que o $\mathrm{p} K_{a}$ do ligante imidazólico estudado encontra-se em $8,5 .{ }^{38}$ Observa-se que em meio ácido há uma degradação do complexo de cobre(II), diminuindo a sensibilidade, mostrando que a hidrólise do ligante influencia na queda de resposta amperométrica, pois sais comuns de cobre(II), tais como $\mathrm{CuCl}_{2}, \mathrm{Cu}\left(\mathrm{NO}_{3}\right)_{2}$, entre outros sais de cobre(II), não resultam numa catálise eficiente para a redução do $\mathrm{H}_{2} \mathrm{O}_{2}$, devido à extrema distorção tetragonal. Já em meio alcalino $(\mathrm{pH} \approx 11)$, observa-se uma diminuição de resposta devido a uma provável quebra da ponte imidazolato pelo ânion hidroxo em solução, favorecendo a formação da espécie dinuclear $\left[\mathrm{LCu}\left(\mu-\mathrm{OH}^{-}\right)_{2} \mathrm{CuL}\right]^{2+}$, dificultando a coordenação do peróxido de hidrogênio ao sítio de coordenação do íon metálico. Outra possibilidade, seria a formação do sal $\mathrm{Cu}(\mathrm{OH})_{2}$, com consequente precipitação e perda da sensibilidade. $\mathrm{O}$ sensor proposto apresentou alta sensibilidade em torno de $360 \mathrm{~mA} \mathrm{~cm}^{-2} \mathrm{~mol}^{-1} \mathrm{~L}$ em $\mathrm{pH}$ 7,0, numa faixa linear de concentração entre 12 a $250 \mathrm{mmol} \mathrm{L}^{-1}$, que é um valor considerável se comparado a outros trabalhos, utilizando Nafion ${ }^{\circledR}$ como membrana de imobilização, na determinação de $\mathrm{H}_{2} \mathrm{O}_{2}$, como um sensor modificado com peroxidase de rabanete, potencial $-250 \mathrm{mV}(\mathrm{Ag} / \mathrm{AgCl}) \mathrm{em} \mathrm{pH}$ 7,0, com sensibilidade de $12,8 \mathrm{nA} \mathrm{cm}^{-2} \mathrm{~mol}^{-1} \mathrm{~L} ;{ }^{39}$ sensor modificado com polivinilpiridina e peroxidase de rabanete, potencial $0 \mathrm{mV}$ (ECS) em pH 7,1, com sensibilidade de $16,3 \times 10^{-7} \mathrm{~A} \mathrm{~cm}^{-2} \mathrm{mmol}^{-1} \mathrm{~L}^{40}$ sensor modificado com polipirrol e filme de polifenilenodiamida, potencial $-700 \mathrm{mV}(\mathrm{Ag} / \mathrm{AgCl})$ em $\mathrm{pH} 7,2$, com sensibilidade de $3,90 \times 10^{-7} \mathrm{~A}$ $\mathrm{cm}^{-2} \mu \mathrm{mol}^{-1} \mathrm{~L}^{; 41}$ outro trabalho descreve um biossensor modificado com hexacianoferrato de cobre(II) que apresenta sensibilidade de $1,23 \mu \mathrm{A} \mathrm{cm}^{-2} \mathrm{mmol}^{-1} \mathrm{~L}^{42}$ entre outros relatados na literatura.

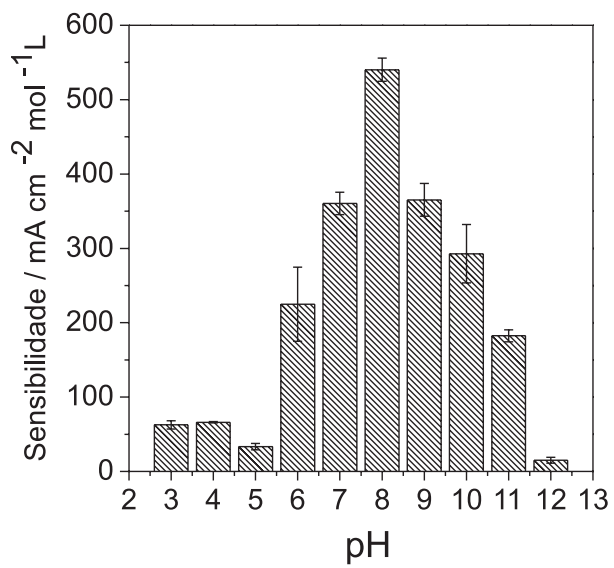

Figura 4. Estudo comparativo de sensibilidades obtidas frente à resposta amperométrica do peróxido de hidrogênio em soluções tampão fosfato $(0,1$ mol $\left.\mathrm{L}^{-1}\right)$ de diversos valores de $\mathrm{pH}$. Medidas obtidas em potencial de -0,50 V

\section{Estudo da influência do $\mathrm{KCl}$ em solução}

Realizou-se também estudo da influência do $\mathrm{KCl}$ na resposta amperométrica frente à detecção do peróxido de hidrogênio. Neste caso, verificou-se uma queda acentuada na sensibilidade do eletrodo modificado na presença de íons cloreto em solução, quando comparado com as mesmas medidas na ausência desses íons, durante 3 medidas consecutivas (Figura $2 \mathrm{~S}$, material suplementar). Uma razão para esse comportamento deve-se ao deslocamento do equilíbrio para formação da espécie dinuclear $\left[\mathrm{LCu}(\mu-\mathrm{Cl})_{2} \mathrm{CuL}\right]^{2+}$, preenchendo o sítio de coordenação do íon metálico e dificultando a coordenação do substrato e sua redução. Desta forma, optamos por usar o eletrólito sem $\mathrm{KCl}$, pois foi a maneira em que obtivemos as melhores respostas.

Estudo da influência da variação do volume de membrana Nafion ${ }^{\circledR}$ e da concentração do complexo de cobre(II) na preparo do eletrodo modificado

Outro estudo realizado foi o da variação do volume de Nafion ${ }^{\circledR}$ adicionado à superfície do ECV, de modo a verificar a sua influência na resposta do sensor. As variações foram de 150, 200 e $250 \mu \mathrm{L}$ de Nafion ${ }^{\circledR} 5 \%$. Com $200 \mu \mathrm{L}$ obtivemos a maior sensibilidade, onde ocorreram também as menores porcentagens de queda de resposta, pois quanto maior a espessura do filme mais difícil será a difusão do analito e o transporte de carga, com consequente queda de sensibilidade, uma vez que a quantidade de íons cobre(II) permaneceu constante nos 3 casos acima (Figura 5).

Realizamos, também, um estudo sobre a influência da concentração do complexo de cobre(II) no filme, variando a massa do com- 


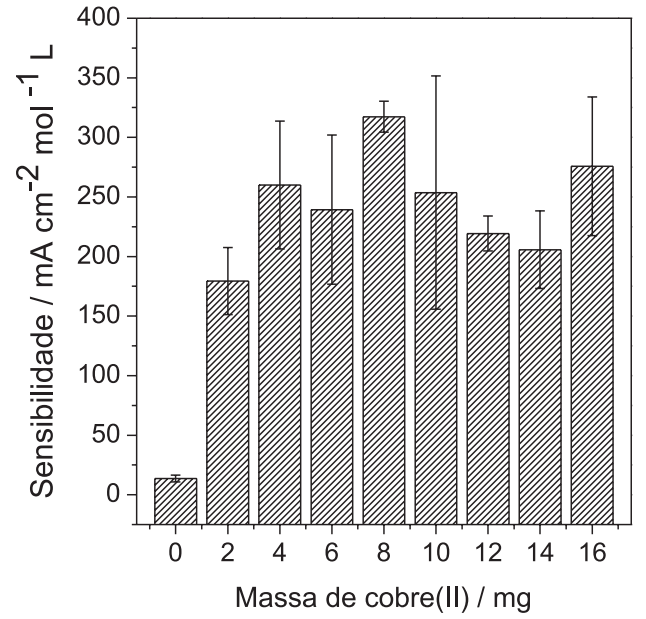

Figura 5. Estudo da influência da porcentagem de massa de cobre(II) na superfície do ECV modificado frente à detecção do peróxido de hidrogênio, mantendo-se o volume de Nafion ${ }^{\circledR}$ constante $(200 \mu L)$

plexo metálico de 0 a $16 \mathrm{mg}$, dissolvido em $200 \mu \mathrm{L}$ de uma solução de Nafion ${ }^{\circledR} 5 \%$ e $1 \mathrm{~mL}$ de água desionizada (Figura 6). As melhores sensibilidades foram obtidas com $8 \mathrm{mg}$ do complexo (1), que também foi a quantidade em que se obteve maior estabilidade do filme sobre a superfície do eletrodo. Diferentemente do que se poderia pensar,

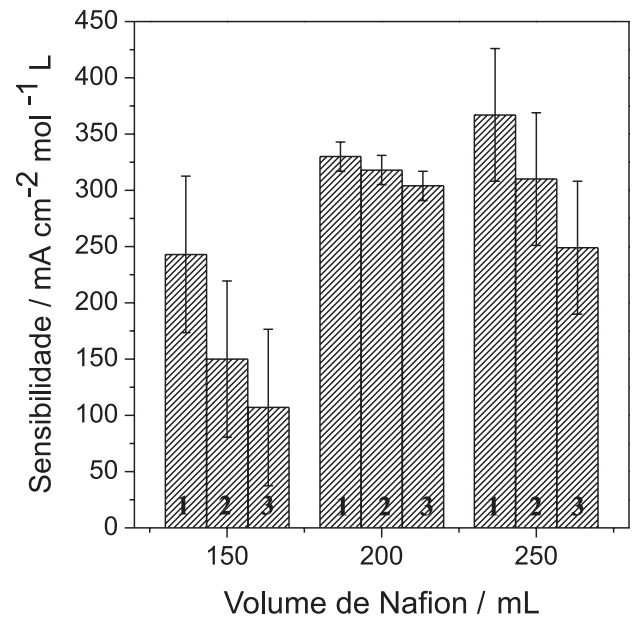

Figura 6. Estudo da influência do volume de Nafion ${ }^{\circledR}$ na superfície do ECV modificado frente à detecção do peróxido de hidrogênio, mantendo-se a quantidade em massa do complexo de cobre(II) constante (8 $\mathrm{mg}$ ) o aumento da quantidade de cobre(II) não necessariamente aumenta a sensibilidade de resposta, pois aumentando a quantidade de íons metálicos e mantendo a camada de Nafion ${ }^{\circledR}$ constante, não há íons sulfonato suficientes para troca com o perclorato do complexo de cobre(II) em excesso, resultando na queda de sensibilidade.

\section{Estudo da influência do ligante ao redor do íon metálico}

O estudo dos filmes híbridos utilizando diferentes complexos de cobre(II) mostrou a obtenção de diferentes valores de sensibilidade, diretamente influenciada pela estrutura do complexo de cobre(II) utilizado e pH do meio para realização das medidas (Tabela 1). As maiores respostas são correspondentes às medidas realizadas com os complexos (1), (3) e (4). As menores respostas são correspondentes aos complexos $(2)$ e $\left[\mathrm{Cu}\left(\mathrm{H}_{2} \mathrm{O}\right)_{6}\right]^{2+}$. Comparando os resultados obtidos entre o composto (1) e as espécies macrocíclicas (3) e (4) é possível notar que, mesmo com uma resposta de sensibilidade mais baixa de (1) em relação às espécies $(3)$ e (4), este sistema possui um menor desvio padrão, indicando maior estabilidade entre as medidas, como observado na Tabela 1.

Uma das possíveis explicações da maior sensibilidade para o complexo (1), quando comparado com os complexos $(2)$ e $\left[\mathrm{Cu}\left(\mathrm{H}_{2} \mathrm{O}\right)_{6}\right]^{2+}$ em pH 7,0 (Tabela 1), se deve ao fato do ligante apyhist promover menor distorção tetragonal na geometria do íon metálico, devido ao efeito Jahn-Teller, favorecendo a coordenação do substrato e transferência eletrônica. De fato, comparando-se os valores da Tabela 1 e Figura $3 \mathrm{~S}$ do material suplementar, verifica-se um valor menor do deslocamento de $g$, dado por $\left[\mathrm{g}_{\mathrm{zz}}-\left(\mathrm{g}_{\mathrm{xx}}+\mathrm{g}_{\mathrm{yy}}\right) / 2\right]$, para o complexo (1) quando comparado às espécies $(2)$ e $\left[\mathrm{Cu}\left(\mathrm{H}_{2} \mathrm{O}\right)_{6}\right]^{2+}$. Além disso, quando se compara a estrutura entre os dois complexos dinucleares (1) e (2), verifica-se uma rombicidade menor para o complexo (1) em relação ao (2), obtida pela diferença entre os parâmetros $\left[\mathrm{g}_{\mathrm{xx}}\right.$ $\mathrm{g}_{\mathrm{yy}}$ ], uma vez que essa diferença é a presença de um grupo $-\mathrm{CH}_{3} \mathrm{e}$ $-\mathrm{H}$ ligado ao grupo funcional imina $(-\mathrm{C}=\mathrm{N}-)$, respectivamente. Este grupamento $-\mathrm{CH}_{3}$ além de promover um menor grau de rombicidade e distorção tetragonal ao redor do íon metálico no complexo (1), ainda pode funcionar como grupo protetor da base de Schiff, dificultando a degradação do ligante durante a reação com $\mathrm{H}_{2} \mathrm{O}_{2}$. Desta forma, verifica-se um menor desvio padrão na resposta para esse complexo, quando comparada ao composto (2), contendo o átomo de $-\mathrm{H}$ ligado ao grupo imina (Esquema 1). De fato, comparando os valores de $\mathrm{A}_{/ /} \mathrm{e}$ $\mathrm{A}_{\perp}$ para os íons de cobre(II) nos compostos (1) e (2), verifica-se uma densidade eletrônica maior sobre o íon metálico para o complexo (1) em relação ao (2), concomitantemente menor densidade eletrônica sobre os nitrogênios complexados (ver valores de $\mathrm{A}_{\mathrm{N}}$ ).

As maiores sensibilidades foram obtidas utilizando os complexos tetranucleares (3) e (4) em solução de pH 9,0. Este arranjo geométrico do catalisador sobre a superfície do eletrodo de carbo-

Tabela 1. Correlação entre as medidas de sensibilidade e parâmetros de RPE para os complexos de cobre(II) estudados

\begin{tabular}{|c|c|c|c|c|c|c|c|c|c|c|}
\hline \multirow{2}{*}{$\begin{array}{l}\text { Compostos de } \\
\text { cobre(II) }\end{array}$} & \multirow[b]{2}{*}{$\mathrm{pH}$} & \multirow{2}{*}{$\begin{array}{c}\text { Sensiblidade } \\
\left(\mathrm{mA} \mathrm{cm}^{2}\right. \\
\left.\mathrm{mol}^{-1} \mathrm{~L}\right)\end{array}$} & \multicolumn{8}{|c|}{ Parâmetros de RPE } \\
\hline & & & $\begin{array}{l}g_{x x} \\
g_{y y}\end{array}$ & $\mathrm{~g}_{\mathrm{zz}}$ & $\begin{array}{c}\mathrm{A}_{/ /} \\
\left(\mathrm{Cu}^{2+} / \mathrm{MHz}\right)\end{array}$ & $\begin{array}{c}\mathrm{A}_{\perp} \\
\left(\mathrm{Cu}^{2+} / \mathrm{MHz}\right)\end{array}$ & $\begin{array}{c}\mathrm{A}_{/ /} \\
(\mathrm{N} / \mathrm{MHz})\end{array}$ & $\begin{array}{c}\mathrm{A}_{\perp} \\
(\mathrm{N} / \mathrm{MHz})\end{array}$ & $\begin{array}{c}{\left[g_{z z}-\left(g_{x x}+g_{y y}\right) / 2\right]} \\
\left(x 10^{-3}\right)\end{array}$ & $\begin{array}{l}{\left[g_{x x}-g_{y y}\right]} \\
\left(x \quad 10^{-3}\right)\end{array}$ \\
\hline $\begin{array}{l}{\left[\mathrm{Cu}\left(\mathrm{H}_{2} \mathrm{O}\right)_{6}\right]^{2+}} \\
\text { Ref. } 43\end{array}$ & 7,0 & $37,0 \pm 11$ & 2,06 & 2,40 & 500 & ---- & ---- & --- & 340,0 & 0 \\
\hline (1) & 7,0 & $360 \pm 13$ & $\begin{array}{l}2,0660 \\
2,0715\end{array}$ & 2,2710 & 520 & 35 & 30 & 44 & 202,2 & 5,5 \\
\hline (2) & 7,0 & $192 \pm 45$ & $\begin{array}{l}2,0570 \\
2.0660\end{array}$ & 2.2690 & 515 & 30 & 37 & 45 & 207,5 & 9,0 \\
\hline (3) & 9,0 & $422 \pm 50$ & \multicolumn{8}{|c|}{ Material pouco solúvel } \\
\hline (4) & 9,0 & $450 \pm 58$ & \multicolumn{8}{|c|}{ Material pouco solúvel } \\
\hline
\end{tabular}

Espectros em metanol/água (4:1, v/v) 
no vítreo parece favorecer a redução do peróxido de hidrogênio (Figura 7), aumentando a sensibilidade deste sistema. Além disso, o desproporcionamento do peróxido de hidrogênio é favorecido em meio alcalino. Em soluções com pH menor que 8, as espécies predominantes são os respectivos aquo-complexos $\left[\mathrm{LCu}\left(\mathrm{H}_{2} \mathrm{O}\right)_{2}\right]^{2+}$ que são menos catalíticos frente à detecção do $\mathrm{H}_{2} \mathrm{O}_{2}$, uma vez que o processo redox necessita de dois elétrons para que ocorra e, nesta estrutura, existe apenas um íon de cobre(II). Estes estudos sugerem que o arranjo molecular influencia na resposta do sensor. Isso pode ser explicado pelo fato da espécie tetranuclear favorecer o reconhecimento molecular do substrato, além disso, a distância entre os íons metálicos é favorecida, permitindo a coordenação e a transferência de dois elétrons ao $\mathrm{H}_{2} \mathrm{O}_{2}$ (Figura 7).

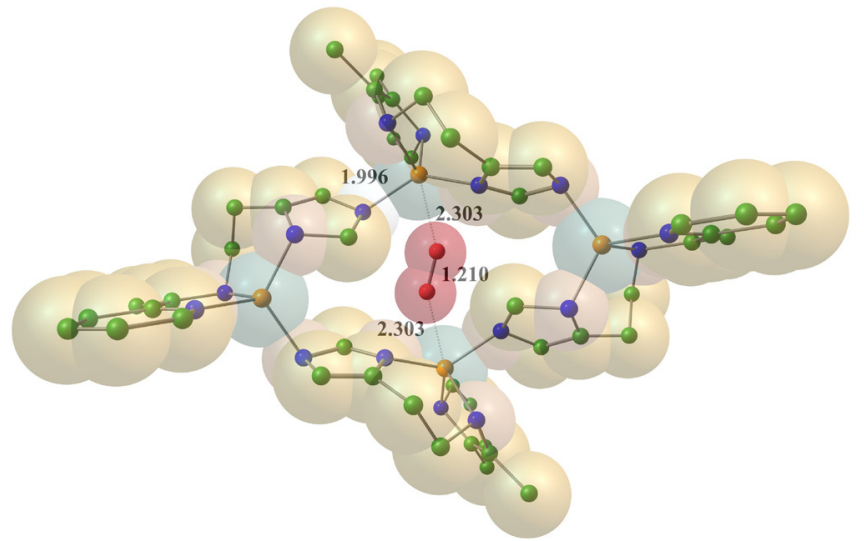

Figura 7. Estrutura otimizada para o complexo (3) para determinação das distâncias entre os íons de cobre(II) e peróxido de hidrogênio (Å). As geometrias foram obtidas utilizando $H F / 3-21 G$

O sensor para detecção de $\mathrm{H}_{2} \mathrm{O}_{2}$ proposto neste trabalho apresenta alto valor de sensibilidade, da ordem de mA, em comparação com os encontrados na literatura, como descrito anteriormente. Porém, o processo biológico de detecção do peróxido de hidrogênio pode ser vulnerável a interferentes, como paracetamol, ácido úrico e ácido ascórbico, substâncias que podem ser facilmente encontradas no organismo humano, devido ao fato desses interferentes possuírem potencial de redução muito próximo ao potencial utilizado na detecção do sensor biomimético apresentado neste trabalho $(\mathrm{E}=-0,50 \mathrm{~V} v s . \mathrm{ECS})$.

\section{CONCLUSÕES}

O sensor amperométrico em desenvolvimento mostra-se bastante promissor na detecção de peróxido de hidrogênio, mostrando boa sensibilidade quando comparado com outros sistemas integrados utilizando diferentes estruturas supramoleculares descritas na literatura. Os resultados obtidos nos estudos de voltametria cíclica para o eletrodo modificado com o complexo (1) mostraram picos de redução e oxidação em $-0,4$ e $-0,3 \mathrm{~V}$, respectivamente, indicando que o sistema possui um comportamento quase reversível e que a etapa limitante deste processo é a difusão do eletrólito até a superfície do eletrodo. O estudo das condições ótimas de trabalho indicou os melhores parâmetros de utilização em $\mathrm{pH}$ 7,0 e potencial -0,50 V ( $v s$. ECS). Uma característica interessante observada foi o fato de haver uma queda acentuada de resposta quando o sistema é colocado na presença de $\mathrm{KCl}$, devido ao preenchimento do sítio de coordenação do íon metálico pelos íons cloreto. Os estudos do volume de membrana Nafion ${ }^{\circledR}$ indicaram um volume ideal para este sistema de $200 \mu \mathrm{L}$ e a quantidade de cobre em $8 \mathrm{mg}$, valores estes que propiciaram valores de sensibilidade mais altos e estáveis. Os estudos realizados até o momento indicam que as espécies tetranucleares de cobre(II) são melhores eletrocatalisadores do que os compostos mono- e dinucleares de cobre(II). Compostos cíclicos parecem facilitar a interação do substrato com o centro metálico. Mantendo-se as mesmas proporções entre os íons de cobre(II) e Nafion ${ }^{\circledR}$, verificou-se que variações no ligante ao redor do íon metálico acarretam mudanças significativas na sensibilidade destas espécies frente à detecção de $\mathrm{H}_{2} \mathrm{O}_{2}$.

\section{MATERIAL SUPLEMENTAR}

O material suplementar está disponível gratuitamente em http:// quimicanova.sbq.org.br, na forma de arquivo PDF.

\section{AGRADECIMENTOS}

À FAPESP (Proc. No. 2008/53576-9; 2009/13785-0), ao CNPq (Proc. No. 474871/2010-0) e ao INCT de Bioanalítica (CNPq, Proc. No. 573672/2008-3 e à FAPESP, Proc. No. 08/57805-2) pelo suporte financeiro. Ao Professor Dr. E. E. Fileti da UNIFESP, São José dos Campos, SP, pelos cálculos teóricos e discussão dos resultados.

\section{REFERÊNCIAS}

1. Kissinger, P. T.; Biosens. Bioelectron. 2005, 20, 2512.

2. Rodriguez-Mozaz, S.; Alda, M. J. L.; Marco, M. -P.; Barceló, D.; Talanta 2005, 65, 291.

3. Freire, R. S.; Pessoa, C. A.; Mello, L. D.; Kubota, L. T.; J. Braz. Chem. Soc. 2003, 14, 230.

4. Reis, R. L.; Curr. Opin. Solid State Mat. Sci. 2003, 7, 263.

5. Sotomayor, M. D. P. T.; Kubota, L. T.; Quim. Nova 2002, 25, 123.

6. Holm, R. H.; Solomon, E. I.; Chem. Rev. 2004, 104, 347.

7. Berg, J. M.; Lippard, S. J.; Curr. Opin. Chem. Biol. 2004, 8, 160.

8. Oliveira, I. R. W. Z.; Osório, R. E.-H. M. B.; Neves, A.; Vieira, I. C.; Sens. Actuators B 2007, 122, 89.

9. Fernandes, S. C.; Osório, R. E-H. M. B.; Anjos, A.; Neves, A.; Micke, G. A.; Vieira, I. C.; J. Braz. Chem. Soc. 2008, 19, 1215.

10. Sotomayor, M. D. P. T.; Tanaka, A. A.; Kubota, L. T.; Electrochim. Acta 2003, 48,855

11. Teixeira, M. F. S.; Segnini, A.; Moraes, F. C.; Marcolino-Júnior, L. H.; Fatibello-Filho, O.; Cavalheiro, E. T. G.; J. Braz. Chem. Soc. 2003, 14, 316.

12. Costa, C. O.; Souza, A. A.; Luz, R. C. S.; Lemos,T. L. G.; Pessoa, O. D. L.; Kubota, L. T.; Goulart, O. F.; J. Braz. Chem. Soc. 2008, 19, 697.

13. Oliveira, I. R. W. Z.; Neves, A.; Vieira, I. C.; Sens. Actuators B 2008 , $129,424$.

14. Franzoi, A. C.; Peralta, R. A.; Neves, A.; Vieira, I. C.; Talanta 2009, 78, 221.

15. Santos, W. J. R.; Sousa, A. L.; Sotomayor, M. D. P. T.; Damos, F. S.; Tanaka, S. M. C. N.; Kubota, L. T.; Tanaka, A. A.; J. Braz. Chem. Soc. 2009, 20, 1180.

16. Gil, E. S.; Kubota, L. T.; J. Braz. Chem. Soc. 2000, 11, 304.

17. Nicholls, P.; Schonbaum, G. R.; The Enzymes, Academic Press: Orlando, 1963, vol. 8, p. 158.

18. Schonbaum, G. R.; Chance, B.; The Enzymes, Academic Press: New York, 1976, vol. 13, p. 363.

19. Zamocky, M.; Koller, F.; Prog. Biophys. Mol. Biol. 1999, 72, 19.

20. Chen, X.; Xie, H.; Kong, J.; Deng, J.; Biosens. Bioelectron. 2001, 16, 115.

21. Fridovich, I.; Annu. Rev. Biochem. 1995, 64, 97.

22. Mattos, I. L.; Shiraishi, K. A.; Braz, A. D.; Fernandes, J. R.; Quim. Nova 2003, 26, 373

23. Janotta, M.; Vogt, F.; Voraberger, H. S.; Waldhauser, W.; Lackner, J. M., Stotter, C.; Beutl, M.; Mizaikoff, B.; Anal. Chem. 2004, 76, 384. 
24. Chai, X. S.; Hou, Q. X.; Luo, Q.; Zhu, J. Y.; Anal. Chim. Acta 2004, 507, 281.

25. Matsubara, C.; Kawamoto, N.; Takamura, K.; Analyst 1992, 117, 1781.

26. Gong, J.; Wang, L.; Zhao, K.; Song, D.; Electrochem. Commun. 2008, $10,123$.

27. Liu, S. Q.; Daí, Z. H.; Chen, H. Y.; Ju, H. X.; Biosens. Bioelectron. 2004, $19,963$.

28. Guascito, M. R.; Filippo, E.; Malitesta, C.; Manno, D.; Serra, A.; Turco, A.; Biosens. Bioelectron. 2008, 24, 1057.

29. Santos, A. S.; Durán, N.; Kubota, L. T.; Electroanalysis 2005, 17, 1103.

30. Jiang, H.-J.; Yang, H.; Akins, D. L.; J. Electroanal. Chem. 2008, 623, 181.

31. Matos, I. O.; Ferreira, T. L.; Paixão, T. R. L. C.; Lima, A. S.; Bertotti, M.; Alves, W. A.; Electrochim. Acta 2010, 55, 5223.

32. Alves, W. A.; Matos, I. O.; Takahashi, P. M.; Bastos, E. L.; Martinho, H.; Ferreira, J. G.; Silva, C. C.; Santos, R. H. A.; Paduan-Filho, A.; Ferreira, A. M. D. C.; Eur. J. Inorg. Chem. 2009, 15, 2219.

33. Alves, W. A.; Cerchiaro, G.; Paduan-Filho, A.; Tomazela, D.M.; Eberlin, M. N.; Ferreira, A. M. D. C.; Inorg. Chim. Acta 2005, 358, 3581

34. Alves, W. A.; Almeida-Filho, S. A.; Santos, R. H. A.; Paduan-Filho, A.; Ferreira, A. M. D. C.; J. Braz. Chem. Soc. 2004, 15, 872.
35. Alves,W. A.; Almeida-Filho, S. A.; Santos, R. H. A.; Ferreira, A. M. D. C.; Inorg. Chem. Commun. 2003, 6, 294.

36. Alves, W. A.; Almeida-Filho, S. A.; Almeida, M. V.; Paduan-Filho, A.; Becerra, C. C.; Ferreira, A. M. D. C.; J. Mol. Catal. A: Chem. 2003, 198, 63.

37. Alves, W. A.; Santos, R. H. A.; Paduan-Filho, A.; Becerra, C. C.; Borin, A. C.; Ferreira, A. M. D. C.; Inorg. Chim. Acta 2004, 357, 2269.

38. Alves, W. A.; Sant'Ana, A. C.; Abbott, M. P.; Homem-de-Mello, P.; Martinho, H.; Santos, R. H. A.; Ferreira, J. G.; Temperini, M. L. A.; Paduan-Filho, A.; Ferreira, A. M. D. C.; Sci. Adv. Mat. 2010, 2, 173.

39. Mohammed, E.; Naranjo-Rodriguez, I.; Temsamani, K. R.; HernándezArtiga, M. P.; Bellido-Milla, D.; Cisneros, J. L. H.-H.; Food Chem. 2008, 110, 1019 .

40. Anh, D. T. V.; Olthuis, W.; Bergveld, P.; Sens. Actuators B 2003, 91, 1.

41. Hamdi, N.; Wang, J.; Monbouquette, H. G.; J. Electroanal. Chem. 2005, $581,258$.

42. Baioni, A. P.; Vidotti, M.; Fiorito, P. A.; Córdoba de Torresi, S. I.; J. Electroanal. Chem. 2008, 622, 219. 


\section{Iorquirene de Oliveira Matos e Wendel A. Alves*}

Centro de Ciências Naturais e Humanas, Universidade Federal do ABC, 05580-970 Santo André - SP / Instituto Nacional de Ciência e Tecnologia de Bioanalítica, CP 6154, 13083-970 Campinas - SP, Brasil

\section{Otaciro R. Nascimento}

Centro de Ciências Naturais e Humanas, Universidade Federal do ABC, 05580-970 Santo André - SP / Departamento de Física e Informática, Instituto de Física de São Carlos, Universidade de São Paulo, 13565-905 São Carlos - SP, Brasil

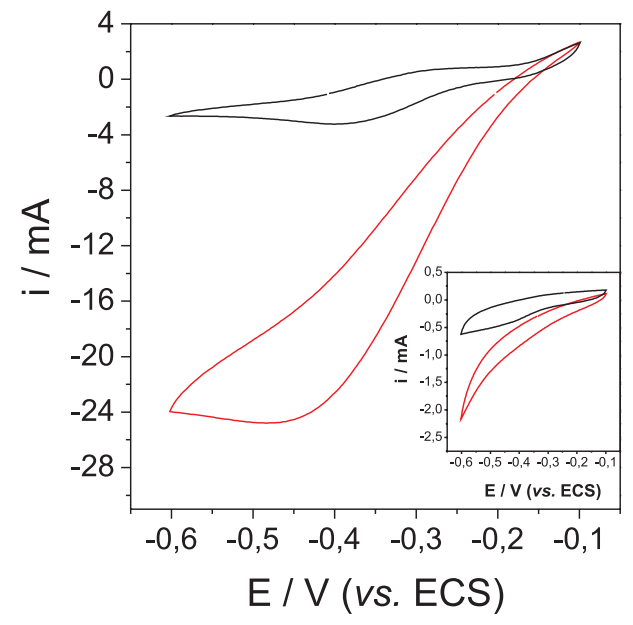

Figura 1S. (A) Estudo do potencial de redução do $\mathrm{H}_{2} \mathrm{O}_{2}$ no ECV modificado sem adição de $\mathrm{H}_{2} \mathrm{O}_{2}$ (preto) e com adição de $500 \mu \mathrm{L}$ de $\mathrm{H}_{2} \mathrm{O}_{2}$ (vermelho). Eletrólito tampão fosfato $\mathrm{pH}$ 7,0 $\left(0,1 \mathrm{~mol} \mathrm{~L}^{-1}\right)$, com velocidade de varredura de $25 \mathrm{mV} \mathrm{s}^{-1}$, após estabilização do filme (20 ciclos), $\mathrm{H}_{2} \mathrm{O}_{2}\left(0,01 \mathrm{~mol} \mathrm{~L}^{-1}\right)$. Gráfico inserido: eletrodo de carbono vítreo, nas mesmas condições descritas acima

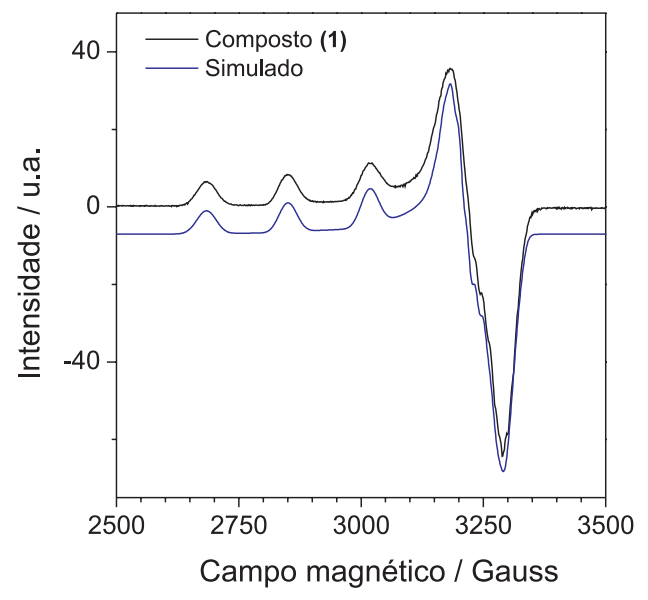

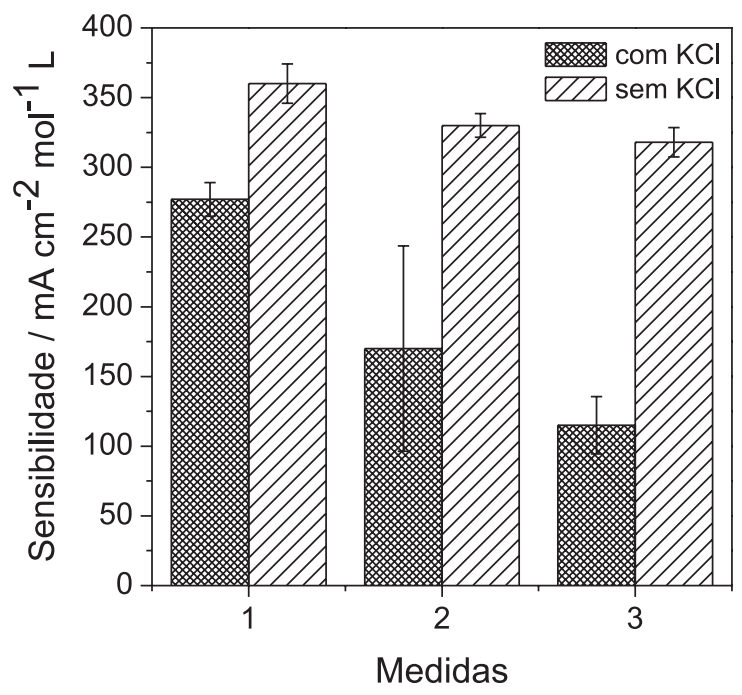

Figura 2S. Estudo da influência dos íons cloreto na resposta amperométrica com adição do peróxido de hidrogênio na solução. Eletrólito tampão fostato pH 7,0 (0,1 mol $\left.\mathrm{L}^{-1}\right)$, potencial aplicado $-0,50 \mathrm{~V}$

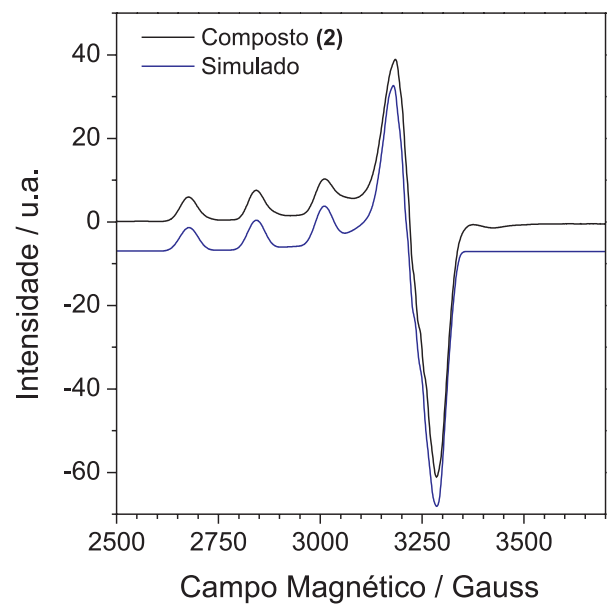

Figura 3S. Espectros RPE experimental e simulado dos compostos (1) e (2) em solução metanol/água (4:1, v/v) a $77 \mathrm{~K}$ 